\title{
Lyapunov Exponents of the Schrödinger Equation with Quasi-Periodic Potential on a Strip
}

\author{
I. Ya. Goldsheid ${ }^{1, \star}$ and E. Sorets ${ }^{2}$ \\ 1 Fakultät für Mathematik, Ruhr-Universität-Bochum (FRG), \\ SFB-237 Bochum-Essen-Düsseldorf, FRG \\ ${ }_{2}$ Institute for Advanced Study, Olden Lane, Princeton, NJ 08540, USA \\ Received June 10, 1991; in revised form September 25, 1991
}

Abstract. We prove that all the non-negative Lyapunov exponents of difference Schrödinger equation

$$
-y_{n+1}+Q_{n} y_{n}-y_{n-1}=0, \quad-\infty<n<+\infty
$$

are strictly positive. Here $y_{n} \in R^{m}$ and $Q_{n}$ is a symmetric $m \times m$ matrix whose offdiagonal elements do not depend on $n$, and the diagonal elements are quasiperiodic functions

$$
q_{n j}(\theta)=\lambda f_{j}\left(e^{2 \pi i(\theta+n \alpha)}\right)-E
$$

with all $f_{i}$ non-constant analytic functions, $\lambda$ sufficiently large, and $\alpha$ any irrational number.

\section{Introduction and Formulation of Results}

In this paper we shall study the Lyapunov exponents of the difference equation:

$$
-y_{n+1}+Q_{n} y_{n}-y_{n-1}=0, \quad-\infty<n<+\infty,
$$

where $y_{n} \in R^{m}$ and $Q_{n}$ is a symmetric $m \times m$ matrix whose off-diagonal elements do not depend on $n$, and the diagonal elements are quasi-periodic functions

$$
q_{n j}(\theta)=\lambda f_{j}\left(e^{2 \pi i(\theta+n \alpha)}\right)-E
$$

with $f_{j}(z)$ non-constant analytic on $\mathscr{A} \equiv\{z|r<| z \mid<1 / r\}$, taking values in $[-1,1]$ for $|z|=1, \lambda$ is a (large) parameter called coupling constant, $E$ is the energy, and $\alpha$ is any irrational number. Without loss of generality we shall assume that $\max _{1 \leqq i \leqq m} \sup _{|z|=1} f_{i}(z)=1$ and $\min _{1 \leqq i \leqq m} \inf _{|z|=1} f_{i}(z)=-1$.

* On leave from Math. Institute, Academy of Sciences USSR, 450057 Ufa, USSR 
Equation (1) becomes equivalent to the finite-difference Schrödinger equation when the off-diagonal elements are chosen properly. For example, the case $q_{i j}=-1$ for $|i-j|=1$ and $q_{i j}=0$ for $|i-j|>1$ corresponds to Schrödinger operator on the strip $\mathbb{Z} \times\{1, \ldots, m\}$. Equation (1) can be written in the form

$$
\left(\begin{array}{c}
y_{n+1} \\
y_{n}
\end{array}\right)=\left(\begin{array}{cc}
Q_{n} & -I \\
I & 0
\end{array}\right)\left(\begin{array}{c}
y_{n} \\
y_{n-1}
\end{array}\right) \equiv A_{n}\left(\begin{array}{c}
y_{n} \\
y_{n-1}
\end{array}\right)
$$

and, thus, the asymptotic behavior of the solutions of Eq. (1) is determined by the asymptotic behavior of the product $S(n):=A_{n} \ldots A_{1}$.

Various problems in solid-state physics give rise to different classes of matrices $Q_{n}$. These classes are characterized by the level of randomness. The case of independent random $Q_{n}$ was studied in [GM1, GM2], where it was shown that under certain algebraic conditions on the support of the corresponding measure in the space of symmetric matrices all the Lyapunov exponents are different and, therefore, the smallest non-negative exponent is, in fact, positive. When $Q_{n}$ 's are non-deterministic at least some of the Lyapunov exponents are strictly positive [K, S, KS]. On the other hand, if $Q_{n}$ 's form a periodic sequence, it is easy to show that the "interesting" Lyapunov exponents vanish.

The case of quasi-periodic potentials exhibits mixed behavior. If the coupling constant $\lambda$ is small and $\alpha$ is poorly approximated by rationals, it is known [BLT] that at least on part of the spectrum the Lyapunov exponent is zero when $m=1$. When $\lambda$ is large and $m=1$ the Lyapunov exponent is positive [Si, CS, FSW, SS].

Until now, quasi-periodic potentials have been studied only for $m=1$ [Si, CS, FSW]. Here we study the case of $m>1$ and large $\lambda$. We do not assume that $\alpha$ is diophantine, only that it is irrational. We shall prove that all non-negative Lyapunov exponents are positive, and, consequently, the Green's function of $H$ decays exponentially for almost every energy $E$. The method we use is an extension of the one used in [SS] which grew out of the analysis of the work of Herman [H].

To describe our result let us consider the following decomposition of $S(n)$ :

$$
S(n)=U(n) D(n) V(n),
$$

where $U, V \in O(n)$, and $D(n)=\operatorname{diag}\left(d_{1}^{(n)}, \ldots, d_{2 m}^{(n)}\right)$ with $d_{1} \geqq d_{2} \geqq \ldots \geqq d_{2 m}>0$. Since $A_{n} \in S p(m, R)$ for all $n$, we have $d_{k}=d_{2 m-k+1}^{-1}$. The $k^{\text {th }}$ Lyapunov exponent $\gamma_{k}$ is defined by

$$
\gamma_{k}:=\lim _{n \rightarrow \infty} \frac{1}{n} \log d_{k}(n) \text {. }
$$

Clearly, $\gamma_{k}=-\gamma_{2 m-k+1}$, and $\gamma_{1} \geqq \gamma_{2} \geqq \ldots \geqq \gamma_{m} \geqq 0$. Existence of the limit in (3) for almost every $\theta$ and its independence of $\theta$ are guaranteed by the Subadditive Ergodic Theorem [Ki] and ergodicity of the underlying dynamical system $\theta \mapsto \theta+\alpha$.

We shall prove the following

Theorem. For $f_{j}$ as above, there exists $\lambda_{0}$ such that for all $\lambda>\lambda_{0}$ and all $E$, there exists a set $\Omega(E) \subset[0,1]$ of Lebesque measure 1 , such that

$$
\gamma_{m}(E)=\gamma_{m}(E, \theta)>0, \quad \forall \theta \in \Omega(E) .
$$

This, together with Oseledeĉ's Multiplicative Ergodic Theorem [O, GM 2] implies existence of $m$ solutions of Eq. (1), which decay exponentially as $n \rightarrow+\infty$ and grow exponentially as $n \rightarrow-\infty$, and $m$ other solutions of Eq. (1), which decay 
exponentially as $n \rightarrow-\infty$ and grow exponentially as $n \rightarrow+\infty$. These $2 m$ solutions are linearly independent and form a basis of all solutions of Eq. (1). There are two important consequences of this fact.

1. The spectrum of the operator $H$ defined by the left-hand side of Eq. (1) is singular.

2. The Green's function of $H$ decays exponentially for almost every energy $E$.

Remarks. We should point out that the spectrum of $H$ can actually be purely singular continuous if $\alpha$ is a Liouville number [CFKS].

The requirement that $\lambda$ is large cannot be avoided, for when $\lambda$ is small (and $m=1)$ KAM theory is applicable and there is absolutely continuous spectrum [BLT].

The reader will see that we very strongly use the non-triviality of $f_{i}$ 's in our proof. This condition is necessary, for there are examples in which the presence of constant $f_{i}$ 's leads to appearance of zero Lyapunov exponents.

\section{Plan of the Proof}

We shall obtain a lower bound on $\gamma_{m}$ by estimating $\sum_{1}^{m} \gamma_{i}$ and $\sum_{1}^{m-1} \gamma_{i}$. Without loss of generality we can assume that $E \in \sigma(H)$, for otherwise Green's function decays exponentially and, consequently, $\gamma_{m}>0$.

Proposition 1. For $E \in \sigma(H)$,

$$
\sum_{1}^{m-1} \gamma_{i} \leqq(m-1) \log 2(\lambda+c)
$$

where $c$ is the norm of the off-diagonal part of $Q_{0}$.

Proposition 2. For $E \in \sigma(H)$,

$$
\sum_{1}^{m} \gamma_{i} \geqq m \log \lambda+\text { const }
$$

Proof of Theorem. Combining Propositions 1 and 2, we have

$$
\gamma_{m}=\sum_{1}^{m} \gamma_{i}-\sum_{1}^{m-1} \gamma_{i} \geqq \log \lambda+\text { const }>0
$$

for $\lambda$ sufficiently large. We note that the constant above depends on $m$, the functions $f_{i}$, and $c$.

\section{Proofs of the Propositions}

Proof of Proposition 1. We first note that

$$
\begin{aligned}
\sum_{1}^{m-1} \gamma_{i} & =\lim _{n \rightarrow \infty} \frac{1}{n} \sum_{1}^{m-1} \log d_{i}(n) \leqq \lim _{n \rightarrow \infty} \frac{1}{n}(m-1) \log d_{1}(n)=\lim _{n \rightarrow \infty} \frac{1}{n}(m-1) \log \|S(n)\| \\
& \leqq(m-1) \sup _{n} \log \left\|A_{n}\right\| \leqq(m-1) \sup _{n} \log \left(\left\|Q_{n}\right\|+2\right) .
\end{aligned}
$$


Now, $\|Q\| \leqq \max _{1 \leqq i \leqq m}\left|\lambda f_{i}-E\right|+c$, where $c$ is the norm of the off-diagonal part of $Q(n)$. Since $E \in \sigma(H) \subset[-\lambda-c, \lambda+c]$,

$$
\left\|Q_{n}\right\| \leqq 2(\lambda+c)
$$

Consequently,

$$
\sum_{1}^{m-1} \gamma_{i} \leqq(m-1)(\log \lambda+\text { const })
$$

Proof of Proposition 2. To estimate $\sum_{1}^{m} \gamma_{i}$ from below we note the following. Let $\pi$ be any $m$-dimensional plane in $R^{2 m}$ and let $\left\{v_{1}, \ldots, v_{m}\right\}$ be its basis. Then

$$
2 \sum_{1}^{m} \gamma_{i} \geqq \lim _{n \rightarrow \infty} \frac{1}{n} \log \left|G\left(S(n) v_{1}, \ldots, S(n) v_{m}\right)\right|,
$$

where $G\left(x_{1}, \ldots, x_{m}\right)$ is, by definition, Gram's determinant of the vectors $x_{1}, \ldots, x_{m}$. Direct investigation of the right-hand side of $(8)$ is rather difficult because it is hard to follow the evolution of the vectors $S(n) v_{i}$ since the diagonal elements of $Q(n)$ can be small. We are going to avoid this problem with the help of the following extension of Jensen's formula [SS].

Lemma 1. Let $g$ be meromorphic on the annulus $\mathscr{A}:=\{z: r<|z|<1\}$. Then

$$
\begin{aligned}
\int_{0}^{1} \log \left|g\left(e^{2 \pi i \theta}\right)\right| d \theta= & \int_{0}^{1} \log \left|g\left(r e^{2 \pi i \theta}\right)\right| d \theta+\sum \log \left|p_{i}\right| \\
& +\sum \log \frac{1}{\left|r_{j}\right|}+\left(\log \frac{1}{r}\right) \underset{|z|=r}{\operatorname{Arg} g},
\end{aligned}
$$

where $p_{i}$ and $r_{j}$ are the poles and the roots of $g$ in $\mathscr{A}$, and

$$
\underset{|z|=r}{\operatorname{Arg} g}=\frac{1}{2 \pi i} \int_{|z|=r} \frac{g^{\prime}}{g} d z .
$$

In our case, $g(z)=G\left(S(n) v_{1}, \ldots, S(n) v_{m}\right)$. We recall that $S(n)$ is a function of $z$, because our potential depends on $z$.

Since $G$ is analytic on $\mathscr{A}$ and $\left|r_{j}\right| \leqq 1$ for all $j$, Jensen's formula leads to the following inequality:

$$
\int_{|z|=1} \log |G| d \theta \geqq \int_{|z|=r} \log |G| d \theta+\left(\log \frac{1}{r}\right) \underset{|z|=r}{\operatorname{Arg} G .}
$$

In order to estimate each of the terms on the right-hand side of $(10)$ we are going to choose $r \in(0,1)$ so that the diagonal elements $q_{n i}(z)$ of $Q_{n}$ satisfy

$$
\left|q_{n i}(z)\right| \geqq \min _{|z|=r}\left|\lambda f_{i}(z)-E\right|>\lambda \delta_{0},
$$

where $\delta_{0}>0$ depends on the functions $f_{i}$ only. To see that such $\delta_{0}$ exists we note that since $f_{i}$ 's are non-constant and analytic, for each $c \in R$ there exist $r(c)$ and $\delta(c)$ so that

$$
\left|f_{i}(z)-c\right|>\delta(c)>0 \text { for }|z|=r(c) \text { and } i=1, \ldots, m
$$

and the union of the ranges of values of $f_{i}$ is compact. 
Lemma 2. $A_{n}(z)$ for $|z|=r$ preserve the set $\mathscr{F}$ of $m$-dimensional planes spanned by vectors of the form

$$
\left(\begin{array}{c}
x \\
\phi x
\end{array}\right)
$$

where $x \in R^{m}$ and the operator $\phi: R^{m} \rightarrow R^{m}$ satisfies $\|\phi\| \leqq 2 /\left(\lambda \delta_{0}\right)$.

Proof. For any $n$,

$$
A_{n}\left(\begin{array}{c}
x \\
\phi x
\end{array}\right)=\left(\begin{array}{c}
\left(Q_{n}-\phi\right) x \\
x
\end{array}\right)=\left(Q_{n}-\phi\right)\left(\begin{array}{c}
x \\
\left(Q_{n}-\phi\right)^{-1} x
\end{array}\right) .
$$

Therefore, if $\phi_{n+1}=\left(Q_{n}-\phi_{n}\right)^{-1}$ it follows from (11) that

$$
\left\|\phi_{n+1}\right\| \leqq \frac{1}{\lambda \delta_{0}-c-\left\|\phi_{n}\right\|}<\frac{2}{\lambda \delta_{0}}
$$

for $\lambda>\lambda_{0}$ for some large $\lambda_{0}$.

Let $\pi_{0}$ be the plane spanned by the vectors of the form

$$
\left(\begin{array}{c}
e_{i} \\
0
\end{array}\right) \in R^{2 m}
$$

where $\left\{e_{1}, \ldots, e_{m}\right\}$ form the standard basis of $R^{m}$ and let $\pi_{n}=A_{n} \ldots A_{1} \pi_{0}$ for $n \geqq 1$.

For any matrix $A$ and plane $\pi$, the dilation coefficient

$$
G(A, \pi):=\frac{G\left(A v_{1}, \ldots, A v_{m}\right)}{G\left(v_{1}, \ldots, v_{m}\right)}
$$

is independent of the choice of the spanning vectors $v_{1}, \ldots, v_{m}$. Consequently,

$$
G\left(S(n) e_{1}, \ldots, S(n) e_{m}\right)=\prod_{i=1}^{n} G\left(A_{i}, \pi_{i-1}\right) .
$$

Remark. For $v_{i} \in \mathbb{C}^{m}$ we define

$$
G\left(v_{1}, \ldots, v_{m}\right):=\operatorname{det}\left(\left(v_{i}, v_{j}\right)\right)_{i, j=1}^{m},
$$

where

$$
\left(v_{i}, v_{j}\right)=\sum_{k=1}^{m} v_{i}^{k} v_{j}^{k}
$$

with $v_{i}^{k}$ being the components of the vectors $v_{i}$. We note that (14) is not actually a scalar product, but defining $(\cdot, \cdot)$ in this way makes the function $G(A, \pi)$ analytic in $z$. We shall make essential use of this fact.

Lemma 3. If $\left\|\phi_{0}\right\| \leqq 2 /\left(\lambda \delta_{0}\right)$, then

$$
G\left(A_{i}(z), \pi_{i-1}\right)=\prod_{k=1}^{m}\left(\lambda f_{k}(z)-E\right)^{2}\left(1+O\left(\frac{1}{\lambda}\right)\right) .
$$

Proof. By Lemma $2,\left\|\phi_{i}\right\| \leqq 2 /\left(\lambda \delta_{0}\right)$ for all $i>0$. Let $e_{1}, \ldots, e_{m}$ be the standard basis. Then the $2 m$-dimensional vectors

$$
v_{k}=\left(\begin{array}{c}
e_{k} \\
\phi_{i-1} e_{k}
\end{array}\right)
$$


span $\pi_{i-1}$, and the dilation coefficient

Now,

$$
G\left(A, \pi_{i-1}\right)=\frac{G\left(A v_{1}, \ldots, A v_{m}\right)}{G\left(v_{1}, \ldots, v_{m}\right)}
$$

$$
A_{i} v_{k}=\left(\begin{array}{c}
\left(Q_{i}-\phi_{i-1}\right) e_{k} \\
e_{k}
\end{array}\right)=\left(\lambda f_{k}-E\right)\left(\begin{array}{c}
e_{k}+O\left(\frac{1}{\lambda}\right) \\
O\left(\frac{1}{\lambda}\right)
\end{array}\right)=:\left(\lambda f_{k}-E\right) h_{k}
$$

It follows that

$$
G\left(A_{i} v_{1}, \ldots, A_{i} v_{m}\right)=\prod_{k=1}^{m}\left(\lambda f_{k}-E\right)^{2} G\left(h_{1}, \ldots, h_{m}\right)=\prod_{k=1}^{m}\left(\lambda f_{k}-E\right)^{2}\left(1+O\left(\frac{1}{\lambda}\right)\right) .
$$

Since $G\left(v_{1}, \ldots, v_{m}\right)=1+O\left(\lambda^{-1}\right)$, Lemma 3 is proved.

\section{Lemma 4.}

$$
\frac{1}{n} \int_{|z|=r} \log \left|G\left(S(n) e_{1}, \ldots, S(n) e_{m}\right)\right| \geqq 2 m \log \lambda \delta_{0}+O\left(\frac{1}{n}\right) .
$$

Proof. Lemma 3, Ineq. (11), and Eq. (13) imply

$$
\log \left|G\left(S(n) e_{1}, \ldots, S(n) e_{m}\right)(z)\right| \geqq n\left(2 m \log \lambda \delta_{0}+O\left(\frac{1}{\lambda}\right)\right) .
$$

\section{Lemma 5.}

$$
\mid \underset{|z|=r}{\operatorname{Arg} G\left(S(n) e_{1}, \ldots, S(n) e_{m}\right) \mid \leqq \text { const } n .}
$$

Proof. By Lemma 3, Eq. (13), and Rouche's theorem

$$
\underset{|z|=r}{\operatorname{Arg}} G=\sum_{i=1}^{n} \sum_{k=1}^{m} \underset{|z|=r}{\operatorname{Arg}}\left(\lambda f_{k}-E\right)^{2}=O(m n) .
$$

Acknowledgements. We would like to thank Thomas Spencer for suggesting the problem and University of California, Irvine for its hospitality. I.G. is also grateful to Courant Institute of Mathematical Sciences, Bochum University, and Rutgers University for their support.

\section{References}

[BLT] Bellisard, J., Lima, R., Testard, D.: A metal-insulator transition for the almost Mathieu model. Commun. Math. Phys. 88, 207-234 (1983)

[CFKS] Cycon, H., Froese, R., Kirsch, W., Simon, B.: Schrödinger operators, with application to quantum mechanics and global geometry. Berlin, Heidelberg, New York: Springer 1987

[CS] Chulaevsky, V., Sinai, Ya.: Anderson localization for multi-frequency quasi-periodic potentials in one dimension. Commun. Math. Phys. 125, 91-112 (1989)

[FSW] Fröhlich, J., Spencer, T., Wittwer, P.: Localization for a class of one-dimensional quasiperiodic potentials. Commun. Math. Phys. 132, 5-25 (1990)

[GM 1] Goldsheid, I.Ya., Margulis, G.A.: Conditions on simplicity of the spectrum of Lyapunov indices. Dokl. Akad. Nauk SSSR 293, 297-301 (1987). Translation in Sov. Math. Dokl. 35, 309-313 (1987) 
[GM 2] Goldsheid, I.Ya., Margulis, G.A.: Lyapunov indices of products of random matrices. Uspekhi Mat. Nauk 44 (5), 13-60 (1989). Translation Russ. Math. Surv. 44 (5), 11-71 (1989)

[H] Herman, M.: Une méthode pour minorer les exposants de Lyapunov et quelques exemples montrant le charactère local d'un Théorème d'Arnold et de Moser sur le tore en dimension 2. Commun. Math. Helv. 58, 453-502 (1983)

[Ki] Kingman, J.F.C.: Subadditive processes. Lecture Notes in Math., vol. 539. Berlin, Heidelberg, New York: Springer 1976

[K] Kotani, S.: Ljapunov indices determine absolutely continuous spectrum of stationary random one-dimensional Schrödinger operators. Proc. of Taniguchi Symp. SA. Katata 225-247 (1982)

[KS] Kotani, S., Simon, B.: Stochastic Schrödinger operators and Jacobi matrices on the strip. Commun. Math. Phys. 119, 403-429 (1988)

[O] Oseledeĉ, V.I.: A multiplicative ergodic theorem, characteristic Lyapunov exponents of dynamical systems. Trudy Moskov. Mat. Obshch. 19, 179-210 (1968). Translation in Trans. Mosc. Math. Soc. 19, 197-231 (1968)

[S] Simon, B.: Kotani theory for one-dimensional stochastic Jacobi matrices. Commun. Math. Phys. 89, 227-234 (1983)

[Si] Sinai, Ya.: Anderson localization for one-dimensional difference Schrödinger operator with quasi-periodic potential. J. Stat. Phys. 46, 861-918 (1987)

[SS] Sorets, E., Spencer, T.: Positive Lyapunov exponents for Schrödinger operator with quasi-periodic potentials. Commun. Math. Phys. 142, 543-566 (1991)

Communicated by T. Spencer 
